\title{
Allogeneic CD19-specific CAR-modified CD8 Plus Central Memory-derived Virus- specific T Cells
}

National Cancer Institute

\section{Source}

National Cancer Institute. Allogeneic CD19-specific CAR-modified CD8 Plus Central

Memory-derived Virus-specific T Cells. NCI Thesaurus. Code C99215.

A preparation of allogeneic Epstein-Barr virus (EBV)- and human cytomegalovirus (CMV)specific CD8+ central memory-derived T effector-lymphocytes that have been genetically modified to express a chimeric antigen receptor (CAR) anti-CD19/CD3 zeta chain fusion protein coupled to the intracellular signal domain of CD28 antigen, with potential immunostimulating, anti-viral and antineoplastic activities. Upon infusion, allogeneic CD19-specific CAR-modified CD8+ central memory-derived virus-specific T cells directs the T-lymphocytes to CD19-expressing tumor cells, stimulating a selective toxicity to tumor cells which may eventually result in tumor cell lysis. CD19 antigen is a Bcell specific cell surface antigen expressed in all B-cell lineage malignancies. The viral specific T-cells exert antiviral immunity. 